\title{
Effect of vitamin D status on clinical pregnancy rates following in vitro fertilization
}

\author{
Kimberley Garbedian MD, Miranda Boggild MD, Joel Moody MD, Kimberly E. Liu MD
}

Abstract

Background: Recent studies suggest that vitamin D may play a role in human reproduction. Our goal was to investigate whether vitamin D levels are predictive of implantation and clinical pregnancy rates in infertile women following in vitro fertilization (IVF).

Methods: We prospectively evaluated vitamin D status, as determined by serum 25-hydroxy-vitamin D (25[OH]D) levels, in a cohort of 173 women undergoing IVF at Mount Sinai Hospital, Toronto, Ontario. Serum 25(OH)D samples were collected within 1 week before oocyte retrieval. We classified patients as having sufficient $(\geq 75 \mathrm{nmol} / \mathrm{L})$ or insufficient (or deficient; hereafter referred to as "insufficient"; < $75 \mathrm{nmol} / \mathrm{L}$ ) serum levels of 25(OH)D. We compared patient demographics and IVF cycle parameters between groups. The primary outcome measure was clinical pregnancy (intrauterine sac visible on ultrasound performed 4-5 weeks after embryo transfer).

Results: Of the included women, $54.9 \%$ had insufficient $25(\mathrm{OH}) \mathrm{D}$ levels and $45.1 \%$ had sufficient levels. Women with sufficient levels had significantly higher rates of clinical pregnancy per IVF cycle started (52.5\%) compared with women with insufficient levels (34.7\%; $p<0.001)$. Implantation rates were also higher in the sufficient 25(OH)D group, but the results were not statistically significant. Multivariable logistic regression analysis (adjusted for age, body mass index and day 5 [v. day 3] embryo transfer) showed that serum 25(OH)D level may be a predictor of clinical pregnancy (adjusted odds ratio 1.01, 95\% confidence interval 1.00-1.03).

Interpretation: Our findings suggest that women with sufficient levels of vitamin D are significantly more likely to achieve clinical pregnancy following IVF. Vitamin D supplementation could provide an easy and cost-effective way of improving pregnancy rates; this merits further investigation. Trial registration: ClinicalTrials.gov, no. NCT01348594.

T nfertility affects $15 \%$ of couples in North America. ${ }^{1}$ Recent studies support the role of vitamin D in human reproduction and suggest that vitamin $\mathrm{D}$ levels predict reproductive success following in vitro fertilization (IVF). ${ }^{2,3}$

Vitamin D is a prohormone that is acquired exogenously from the diet or produced endogenously in the skin. Vitamin $\mathrm{D}$ is metabolized primarily in the liver to 25 -hydroxy-vitamin $\mathrm{D}(25[\mathrm{OH}] \mathrm{D})$, the serum concentration of which can be used as an indicator of vitamin D status. The classification of vitamin D status varies in the literature, ${ }^{2,4,5}$ and because the relation between vitamin $\mathrm{D}$ and fertility has only recently been investigated, no specific cut-off values have been referenced in the literature. However, a Canadian guideline defined vitamin D deficiency as levels below $25 \mathrm{nmol} / \mathrm{L}$, insufficiency as levels between 25 and $74 \mathrm{nmol} / \mathrm{L}$, and sufficiency as levels of 75 $\mathrm{nmol} / \mathrm{L}$ and greater. ${ }^{6}$ Lower levels of vitamin $\mathrm{D}(<75 \mathrm{nmol} / \mathrm{L})$ have also been associated with a higher incidence of certain types of cancer and impaired immune response. ${ }^{4.5}$

People living in countries at higher latitudes, such as the United States and Canada, are more prone to vitamin D insufficiency, especially during the winter months. ${ }^{7}$ Vitamin D insufficiency is highly prevalent in women of reproductive age. ${ }^{2,7}$ A Canadian study by Veith and colleagues ${ }^{7}$ reported that
$25.6 \%$ of nonwhite and $14.8 \%$ of white women of reproductive age (18-35 yr) had insufficient vitamin D levels (defined as $<40 \mathrm{nmol} / \mathrm{L})$. Ozkan and colleagues ${ }^{2}$ reported a $36 \%$ prevalence of vitamin D insufficiency $(50-74 \mathrm{nmol} / \mathrm{L})$ and a $27 \%$ prevalence of deficiency $(<50 \mathrm{nmol} / \mathrm{L})$ among women of reproductive age with infertility. ${ }^{2}$ Anifandis and colleagues ${ }^{8}$ reported that the prevalence of vitamin D insufficiency (20.1$30 \mathrm{ng} / \mathrm{mL})$ or deficiency $(<20 \mathrm{ng} / \mathrm{mL})$ was $79 \%$ in a population of women undergoing IVF.

The link between vitamin $\mathrm{D}$ status and reproduction has been largely shown in murine models. ${ }^{9-12}$ In an early study by Halloran and Deluca, ${ }^{9}$ female rats were fed diets that were sufficient or deficient in vitamin $\mathrm{D}$. The rats were then mated

Competing interests: Kimberley Garbedian and Kimberly Liu hold an unrestricted educational grant from Ferring Canada for a separate clinical study. No competing interests declared by Joel Moody and Miranda Boggild.

This article has been peer reviewed.

Correspondence to: Kimberley Garbedian, kliu@mtsinai.on.ca CMAJ Open 2013.DOI:10.9778/cmajo.20120032 
and their fertility determined. The authors reported that rats fed the diet deficient in vitamin $\mathrm{D}$ had $75 \%$ reduced fertility and $30 \%$ smaller litter sizes than rats fed the sufficient diet.' Hickey and colleagues ${ }^{10}$ also found that female rats fed a diet lacking vitamin D before mating had significantly smaller litters than rats fed a diet containing vitamin $\mathrm{D}$.

Further evidence supporting an association between vitamin D and reproduction comes from studies of the vitamin D receptor. ${ }^{12-14}$ Vitamin D receptors are found in various reproductive tissues, including ovarian and uterine tissue..$^{12,13,15}$ Studies by Yoshizawa and colleagues ${ }^{12}$ observed normal growth and development before weaning (analogous to human puberty) in mice lacking the vitamin $\mathrm{D}$ receptor; however, after weaning, these mice failed to thrive and were infertile. Vitamin D has also been shown to regulate expression of the HOX gene in the uterus. ${ }^{13,14}$ Vitamin D and the transcription factors produced from the HOX genes, specifically HOXA10/11, are hypothesized to function as part of the endocrine signal-transduction pathway, regulating endometrial development in preparation for implantation. ${ }^{13,14}$

Previous studies have examined the effect of vitamin D deficiency on reproductive success following IVF in humans. ${ }^{2,3,816}$ Ozkan and colleagues ${ }^{2}$ performed a prospective cohort study that measured the level of $25(\mathrm{OH}) \mathrm{D}$ in the follicular fluid of 84 women undergoing IVF. They found that women with higher levels of vitamin D in their follicular fluid were significantly more likely to achieve implantation and clinical pregnancy following IVF. ${ }^{2}$ A subsequent retrospective analysis of vitamin D levels in oocyte donor and recipient cycles (i.e., the recipient receives an embryo derived from an oocyte donor), by Rudick and colleagues ${ }^{3}$ also found that vitamin D insufficiency in the oocyte recipients was associated with lower rates of clinical pregnancy. Conversely, a small prospective study by Aleyasin and colleagues ${ }^{16}$ did not find a significant difference in biochemical (i.e., very early pregnancy loss) or clinical pregnancy rates across tertiles of follicular fluid 25(OH)D levels. ${ }^{16} \mathrm{~A}$ prospective cohort study by Anifandis and colleagues ${ }^{8}$ suggested that higher $25(\mathrm{OH}) \mathrm{D}$ levels, combined with decreased glucose levels, in follicular fluid may have a negative impact on the success of IVF. In this study, the rate of clinical pregnancy was $32.3 \%$ in the $25(\mathrm{OH}) \mathrm{D}$ deficient group $(<20 \mathrm{ng} / \mathrm{mL}), 32.7 \%$ in the insufficient group (20.1$30 \mathrm{ng} / \mathrm{mL}$ ) and $14.5 \%$ in the sufficient group (> $30 \mathrm{ng} / \mathrm{mL})$ $(p=0.047)$.

In the current study, our goal was to determine whether serum vitamin D levels are predictive of IVF outcomes among infertile women. Our main objective was to investigate whether vitamin $\mathrm{D}$ deficiency is associated with lower rates of clinical pregnancy after IVF.

\section{Methods}

\section{Study design}

We prospectively evaluated vitamin $\mathrm{D}$ status, as determined by serum $25(\mathrm{OH}) \mathrm{D}$ levels, in a cohort of women undergoing IVF at Mount Sinai Hospital between April 2011 and
November 2011. The study was approved by our institution's research ethics board, and informed written consent was obtained from all participants. This study was conducted at an academic hospital-based fertility centre.

\section{Patients}

Women undergoing IVF for any indication were eligible for inclusion in this study. Women who met the following criteria were included: aged 18-41 years, follicle-stimulating hormone level $12 \mathrm{IU} / \mathrm{L}$ or lower (baseline level, cycle day 3 ) and able to provide informed consent. We excluded third-party reproduction cycles (i.e., using donor oocytes or a gestational carrier), those with known uncorrected congenital or acquired uterine anomalies, and those who were unable to provide informed consent (e.g., language barrier).

Demographic data and IVF cycle data for each included patient were obtained via chart review.

\section{IVF cycles}

The included patients underwent IVF cycles as per standard clinical care. Standard agonist (long luteal and microdose flare) protocols and antagonist IVF protocols were used. The agonist protocols used buserelin acetate (Suprefact, Sanofi Aventis; subcutaneous) starting in the midluteal phase at a dose of $0.5 \mathrm{mg} / \mathrm{d}$ for long protocols or at the start of the cycle for microdose flare protocols. The antagonist cycles used cetrolix acetate (Cetrotide, EMD Serono) or ganirelix acetate (Merck), started as per flexible start protocol, when estradiol levels were $2000 \mathrm{pmol} / \mathrm{L}$ or higher or the size of the dominant follicle was $14 \mathrm{~mm}$ or larger. Recombinant or purified urinary follicle-stimulating hormone, with or without lutenizing hormone or human menopausal gonadotropin, was used for controlled ovarian hyperstimulation; the starting doses were determined before study recruitment based on age, folliclestimulating hormone level in the early follicular phase, and the number of antral follicles. Doses were increased or decreased on an individual basis. Ovarian response was assessed by serial transvaginal ultrasonograpy and by serum lutenizing hormone and estradiol assays.

Serum 25(OH)D samples were collected before oocyte retrieval. Nuclear maturation was triggered with $10000 \mathrm{IU}$ of human chorionic gonadotropin (Merck) when 3 or more dominant follicles $(\geq 17 \mathrm{~mm}$ ) were achieved. Transvaginal ultrasound-guided oocyte retrieval was performed 36-38 hours following injection of human chorionic gonadotropin. Ultrasound-guided fresh embryo transfer was performed on day 3 or 5 after fertilization. Vaginal micronized progesterone suppositories were used for luteal-phase support and were continued until fetal cardiac activity was documented.

\section{Outcomes}

The primary outcome was the clinical pregnancy rate per IVF cycle started among women with sufficient $(\geq 75 \mathrm{nmol} / \mathrm{L})$ or insufficient $(<75 \mathrm{nmol} / \mathrm{L})$ vitamin D levels. Clinical pregnancy was defined as an intrauterine sac visible on ultrasound.

The secondary outcomes were prevalence of vitamin D sufficiency, insufficiency and deficiency and the effect of vita- 
min D on IVF cycle parameters (i.e., number of oocytes and implantation rate). Implantation was determined by the presence of a gestational sac, visible by ultrasonography. The implantation rate was calculated as the number of gestational sacs observed by ultrasonography divided by the number of embryos transferred, multiplied by 100 .

\section{Statistical analysis}

Patients were classified as having sufficient $(\geq 75 \mathrm{nmol} / \mathrm{L})$ or insufficient (<75 nmol/L) 25(OH)D levels. Continuous variables are reported as mean ( \pm standard deviation [SD]), and categorical variables are reported as percentages. We used $\chi^{2}$ and Student $t$ tests or Mann-Whitney $\mathrm{U}$ tests to analyze categorical and continuous variables, respectively. We used multivariable logistic regression to evaluate the relation between serum 25(OH)D level and implantation and clinical pregnancy after adjustment for parameters known to influence the IVF success (age, body mass index [BMI], day 5 [v. day 3] embryo transfer). (At our institution, embryo transfer is performed on day 5 if there are at least 5-6 good quality embryos present on day 3.) We calculated sample size $(n=170)$ using a power of 0.8 , a significance level of 0.05 , and a $95 \%$ confidence interval (CI). We expected the clinical pregnancy rate to be $40 \%$ among women with sufficient vitamin $\mathrm{D}$ levels and $20 \%$ among women with insufficient levels, based on our institution's clinical pregnancy rates and previous vitamin D IVF studies. ${ }^{2}$

All data analyses were performed using IBM SPSS Statistics version 19.0 .

\section{Results}

We recruited 182 patients, and 173 were included in our analysis. We excluded 9 women because they did not meet our inclusion criteria: 4 women aged greater than 41 years, and 5 women who did not start an IVF cycle. All of the included patients underwent oocyte retrieval, and 162 underwent embryo transfer. Of the 11 patients who did not undergo embryo transfer, 4 women did not have oocytes or embryos of sufficient quality, and 7 had their embryos frozen secondary to ovarian hyperstimulation syndrome $(n=3)$, fertility preservation $(n=1)$, hydrosalpinx $(n=1)$, no sperm available $(n=1)$ or inappropriate endometrial lining $(n=1)$. There was no difference in the distribution of these women between insufficient and sufficient groups.

The prevalence of vitamin D deficiency, insufficiency and sufficiency was $1.2 \%, 53.8 \%$ and $45.1 \%$, respectively. In our analyses, we grouped together women with deficient and insufficient 25(OH)D levels (hereafter referred to as "insufficient"). Body mass index was significantly higher among women with insufficient vitamin D levels (mean $24.8 \pm$ SD 4.7) than among women with sufficient levels (mean $23.3 \pm$ SD 3.8; $p=0.02$ ) (Table 1). The remaining patient characteristics did not differ significantly between groups. Table 2 shows the IVF cycle parameters for women in both groups. Those in the sufficient $25(\mathrm{OH}) \mathrm{D}$ group were more like to undergo embryo transfer on day 5 (v. day $3 ; 71.8 \%$ ) compared to women in the insufficient group $(58.9 \%, p=0.054)$. There was no difference in the number of oocytes retrieved, the percentage of cycles in which intracytoplasmic sperm injection was performed and number of embryos transferred.

We found a higher clinical pregnancy rate per IVF cycle started among women with sufficient $25(\mathrm{OH}) \mathrm{D}$ levels $(52.5 \%)$ than among women with insufficient levels $(34.7 \%$; $p$ $<0.001$ ) (Table 2). We also observed a higher clinical pregnancy rate per embryo transfer performed among women in the sufficient group than among women in the insufficient group $(54.7 \%$ v. $37.9 \%, p<0.001)$. Although the implantation rate was higher in the sufficient group $(34.5 \%)$ than in the insufficient group (25.6\%), this difference was not statistically significant $(p=0.6)$.

Multivariable logistic regression analysis (adjusted for age, BMI and day 5 [v. day 3] embryo transfer) showed that serum 25(OH)D level was an independent predictor of clinical pregnancy $(p=0.046$; Table 3$)$. Increasing clinical pregnancy rates were observed across serum 25(OH)D tertiles (data not shown).

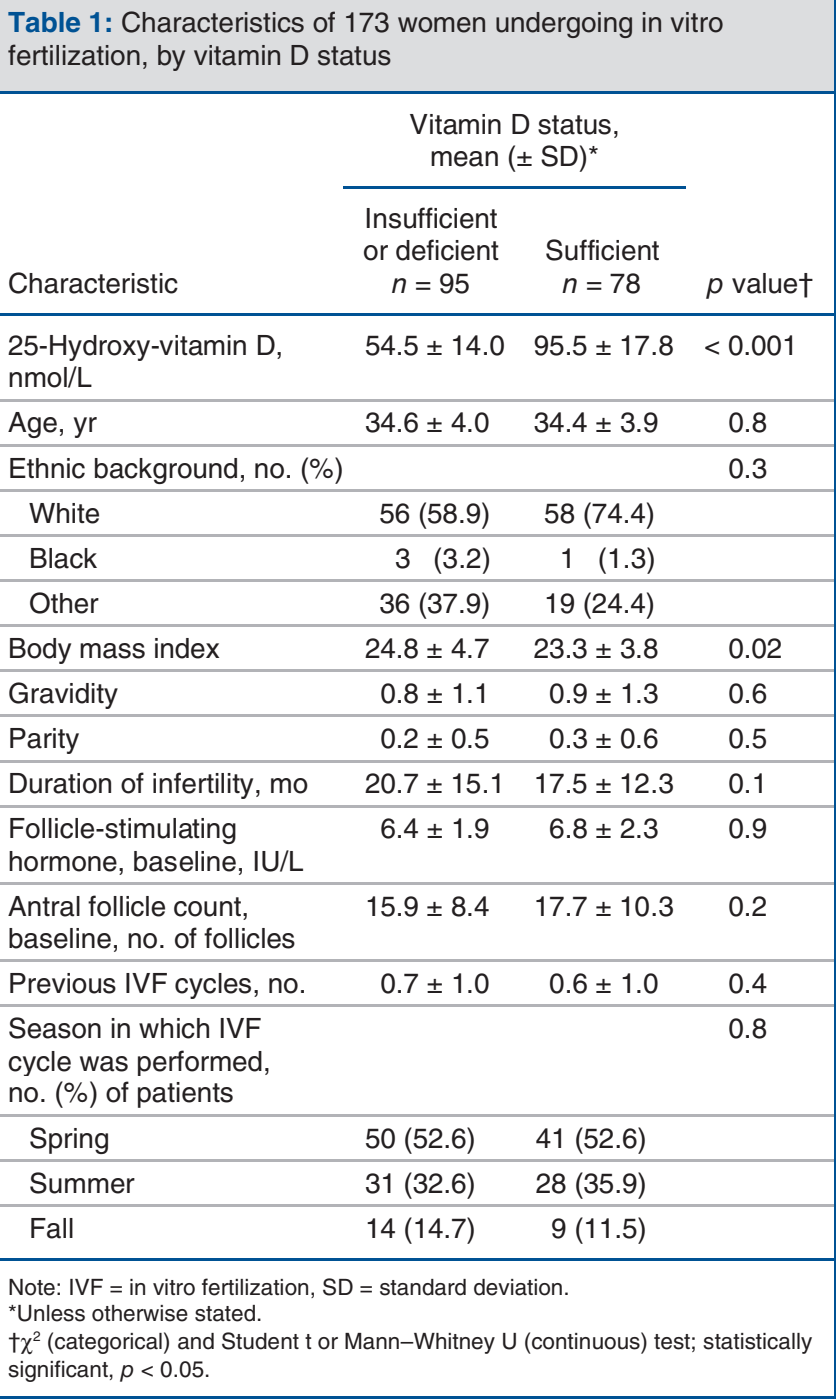




\section{OPEN}

Research

\section{Interpretation}

We found that the serum level of $25(\mathrm{OH}) \mathrm{D}$, as a marker of vitamin $\mathrm{D}$ level, may be a predictor of IVF pregnancy among women undergoing treatment for infertility. Women in our cohort with sufficient vitamin D levels had significantly higher rates of clinical pregnancy following IVF compared with women with insufficient or deficient levels. This finding is clinically significant and may hold potential therapeutic implications because $54.9 \%$ of women in our study had insufficient or deficient levels.

The prevalence of vitamin $\mathrm{D}$ deficiency $(<25 \mathrm{nmol} / \mathrm{L})$, insufficiency $(25-74 \mathrm{nmol} / \mathrm{L})$ and sufficiency $(\geq 75 \mathrm{nmol} / \mathrm{L})$ in our population was $1.2 \%, 53.8 \%$ and $45.1 \%$, respectively. These findings are similar to those previously reported in a North American cohort of reproductive-age women. ${ }^{7}$ The study by Ozkan and colleagues, ${ }^{2}$ which included women of reproductive age with infertility, found a slightly higher preva-

Table 2: In vitro fertilization cycle parameters for 173 women, by vitamin D status

\begin{tabular}{|c|c|c|c|}
\hline \multirow[b]{2}{*}{ Characteristic } & \multicolumn{2}{|c|}{ Vitamin D status, no. (\%) or mean \pm SD } & \multirow[b]{2}{*}{$p$ value } \\
\hline & $\begin{array}{l}\text { Insufficient or deficient } \\
\qquad n=95\end{array}$ & $\begin{array}{c}\text { Sufficient } \\
n=78\end{array}$ & \\
\hline IVF protocol, no. (\%) & & & 0.08 \\
\hline Long & $29(30.5)$ & $34(43.6)$ & \\
\hline Antagonist & $64(67.4)$ & $40(51.3)$ & \\
\hline Flare & $2(2.1)$ & $4(5.1)$ & \\
\hline Day of hCG injection & $12.1 \pm 1.3$ & $11.9 \pm 1.4$ & 0.4 \\
\hline Gonadotropin dose, IU & $2602.8 \pm 1287.3$ & $2547.1 \pm 1330.3$ & 0.8 \\
\hline Follicle diameter > 14 mm, no. & $11.9 \pm 6.5$ & $11.7 \pm 5.8$ & 0.8 \\
\hline Estradiol level on the day of $\mathrm{hCG}$ administration, $\mathrm{pmol} / \mathrm{L}$ & $8556.7 \pm 6187.3$ & $9776.15 \pm 5902.6$ & 0.2 \\
\hline Endometrial thickness on the day of hCG administration, $\mathrm{cm}$ & $1.0 \pm 1.6$ & $1.0 \pm 0.2$ & 0.5 \\
\hline Oocytes retrieved, no. & $12.6 \pm 7.4$ & $12.7 \pm 6.6$ & 0.9 \\
\hline Intracytoplasmic sperm injection, no. (\%) & $74(77.9)$ & $63(80.8)$ & 0.4 \\
\hline Embryos transferred, no. & $1.8 \pm 1.0$ & $1.8 \pm 0.9$ & 0.9 \\
\hline \multicolumn{4}{|l|}{ Day of embryo transfer, no. (\%) } \\
\hline Day 3 & $31(32.6)$ & $19(24.4)$ & 0.2 \\
\hline Day 5 & $56(58.9)$ & $56(71.8)$ & 0.054 \\
\hline Implantation rate, $\%$ & 25.6 & 34.5 & 0.6 \\
\hline Clinical pregnancy rate, per cycle start, $\%$ & 34.7 & 52.5 & $<0.001$ \\
\hline Clinical pregnancy rate, per embryo transfer, \% & 37.9 & 54.7 & $<0.001$ \\
\hline
\end{tabular}

Table 3: Predictors of clinical pregnancy among 173 women undergoing in vitro fertilization

\begin{tabular}{|c|c|c|c|c|}
\hline \multirow[b]{2}{*}{ Variable } & \multicolumn{2}{|c|}{ Crude } & \multicolumn{2}{|c|}{ Adjusted } \\
\hline & $\begin{array}{c}\text { Odds ratio } \\
\text { (95\% confidence interval) }\end{array}$ & $p$ value & $\begin{array}{c}\text { Odds ratio } \\
\text { (95\% confidence interval) }\end{array}$ & $p$ value \\
\hline Age, yr & $0.96(0.89-1.04)$ & 0.3 & $0.98(0.90-1.07)$ & 0.7 \\
\hline Body mass index & $0.92(0.86-1.00)$ & 0.05 & $0.94(0.87-1.01)$ & 0.1 \\
\hline $\begin{array}{l}\text { Day } 5 \text { embryos transferred } \\
\text { (v. day 3) }\end{array}$ & $1.50(0.81-2.90)$ & 0.2 & $1.28(0.61-2.77)$ & 0.5 \\
\hline $\begin{array}{l}\text { 25-Hydroxy-vitamin D, } \\
\mathrm{mmol} / \mathrm{L}\end{array}$ & $1.02(1.00-1.03)$ & 0.2 & $1.01(1.00-1.03)$ & 0.046 \\
\hline
\end{tabular}


lence of vitamin D insufficiency (50-74 nmol/L; 36\%) and deficiency $(<50 \mathrm{nmol} / \mathrm{L} ; 27 \%)$. There was a very low prevalence of vitamin $\mathrm{D}$ deficiency $(<25 \mathrm{nmol} / \mathrm{L})$ in our study $(1.2 \%)$. This may have been because of a difference in our definition of deficiency, because most women in our study were taking prenatal vitamins (400 IU vitamin D) or because our study did not extend over the winter months. However, the percentage of women with insufficient or deficient levels compared to those with sufficient levels did not significantly differ in the spring, summer or fall months or with respect to ethnic background (Table 1).

Body mass index was significantly higher among women in the insufficient $25(\mathrm{OH}) \mathrm{D}$ group (mean 24.8 [ \pm SD 4.7]) than among those in the sufficient group (23.3 $[ \pm$ SD 3.8]; $p=$ $0.02)$. This finding is consistent with existing knowledge about vitamin $\mathrm{D}$ metabolism and has been reported in the literature. ${ }^{17}$ Vitamin $\mathrm{D}$ is a fat soluble vitamin, and adipose tissue is hypothesized to act as a reservoir for its storage, thus reducing its bioavailability. ${ }^{17}$ Lagunova and colleagues ${ }^{17}$ found a significant decrease in serum $25(\mathrm{OH}) \mathrm{D}$ levels in women as $\mathrm{BMI}$ increased. The prevalence of vitamin $\mathrm{D}$ deficiency $(\leq 50 \mathrm{nmol} / \mathrm{L})$ in their study was highest among women with a BMI of 40 or greater.

The mechanism by which vitamin D affects fertility is unclear. Postulated mechanisms include its effect on ovarian steroidogenesis and implantation. ${ }^{2,3,8,13,14} \mathrm{We}$ found no significant differences between women in the sufficient and insufficient $25(\mathrm{OH}) \mathrm{D}$ groups with respect to IVF cycle parameters including the day of human chorionic gonadotropin injection, gonadotropin dose, estradiol level on the day of human chorionic gonadotropin administration, endometrial thickness, the number of oocytes retrieved, or the number of embryos transferred. Therefore, it is unlikely that the observed difference in clinical pregnancy rates was caused by differences in ovarian steroidogenesis. The implantation rate was nonsignificantly higher among women with sufficient $25(\mathrm{OH}) \mathrm{D}$ levels $(p=$ $0.6)$; however, the rate of clinical pregnancy per embryo transfer was significantly higher among women with sufficient levels $(p<0.001)$. One explanation for this finding may be that our study may have been underpowered to detect a difference in the implantation rate. We did find, however, that women with sufficient vitamin D levels were significantly more likely to have a day 5 embryo transfer. At our institution, the decision to transfer an embryo on day 3 versus day 5 is individualized and is based on a number of factors, including the number of good quality embryos. Therefore, the observed difference in the rate of clinical pregnancy between groups may be related to higher embryo quality among women with sufficient $25(\mathrm{OH}) \mathrm{D}$ levels. These results are in contrast to those of Anifandis and colleagues, ${ }^{8}$ who found a higher clinical pregnancy rate among women with deficient or insufficient $25(\mathrm{OH}) \mathrm{D}$ levels in the follicular fluid, suggesting that excess vitamin $\mathrm{D}$ in follicular fluid may negatively affect IVF success. In their study, the mean embryo quality score, but not the cumulative embryo score, was significantly lower among women with sufficient vitamin D levels compared with those in the insufficient and deficient groups $(p=0.009) .{ }^{8}$ Our prospective study is larger than the previously cited studies of the association between vitamin D and IVF outcomes, and we focused on serum (not follicular fluid) levels of vitamin D. Although BMI and the number of day 5 embryo transfers was significantly different between groups, only serum $25(\mathrm{OH}) \mathrm{D}$ level was an independent predictor of clinical pregnancy in our multivariable logistic regression analysis.

\section{Limitations}

This is the largest study in the literature with respect to the relation between vitamin $\mathrm{D}$ and infertility in human IVF populations; however, the interpretation is still limited by the sample size. A larger sample size could have perhaps resulted in a smaller $p$ value and the $95 \%$ CIs may not have included 1 . In this study, we focused on serum vitamin $\mathrm{D}$ levels and did not measure the level of $25(\mathrm{OH}) \mathrm{D}$ in follicular fluid. However, previous studies have shown that the levels in these fluids are highly correlated, ${ }^{2}$ and serum levels have greater clinical utility. If embryo quality scores were available, we would have been better able to determine whether endometrial or embryo quality affected implantation and clinical pregnancy rates. Future studies should focus on determining the mechanism by which vitamin $\mathrm{D}$ affects clinical pregnancy, and they should include measures of embryo quality, implantation and uterine receptivity. Studies should also be undertaken to investigate whether vitamin $\mathrm{D}$ supplementation can improve the rates of pregnancy following IVF.

\section{Conclusion}

Our findings suggest that women with sufficient levels of vitamin D are significantly more likely than those with insufficient levels to achieve clinical pregnancy following IVF. Vitamin D supplementation may be an easy and cost-effective way of improving pregnancy rates and merits further investigation. There was a high prevalence of vitamin D insufficiency or deficiency in our study population. Therefore, it may be beneficial to determine vitamin D status as part of routine infertility assessment and before artificial reproductive treatment, especially in women with higher BMI.

\section{References}

1. Fritz MA, Speroff L. Clinical gynecologic endocrinology and infertility. 8th ed. Philadelphia (PA): Wolters Kluwer Health/Lippincott Williams \& Wilkins; 2011:1137.

2. Ozkan S, Jindal SD, Greenseid K, et al. Replete vitamin D stores predict reproductive success following in vitro fertilization. Fertil Steril 2010;94:1314-9.

3. Rudick B, Ingles SA, Stanczyk K, et al. The role of vitamin D levels on IVF outcomes in donor-recipient cycles. Fertil Steril 2011;95:S8.

4. Holick MF. Vitamin D deficiency. N Engl f Med 2007;357:266-81.

5. Bikle DD. Vitamin D: newly discovered actions require reconsideration of physiological requirements. Trends Endocrinol Metab 2010;21:375-84.

6. Hanley DA, Cranney A, Jones G, et al. Vitamin D in adult health and disease: a review and guideline statement from Osteoporosis Canada. CMA7 2010;182: E610-18.

7. Vieth R, Cole DE, Hawker GA, et al. Wintertime vitamin D insufficiency is common in young Canadian women, and their vitamin D intake does not prevent it. Eur J Clin Nutr 2001;55:1091-7.

8. Anifandis GM, Dafopoulos K. Messini et al. Prognostic value of follicular fluid 25OH vitamin D and glucose levels in the IVF outcome. Repro Biol Endo 2010;8:91.

9. Halloran BP, Deluca HF. Effect of vitamin D deficiency on fertility and reproductive capacity in the female rat. F Nutr 1980;110:1573-80.

10. Hickie JP, Lavigne DM, Woodward WD. Reduced fecundity of vitamin D deficient rats. Comp Biochem Physiol A Comp Physiol 1983;74:923-5.

11. Kwiecinski GG, Petrie GI, DeLuca HF. 1,25-Dihydroxyvitamin D3 restores fertility of vitamin D-deficient female rats. Am 7 Physiol 1989;256:E483-7. 
12. Yoshizawa T, Handa Y, Uematsu Y, et al. Mice lacking the vitamin D receptor exhibit impaired bone formation, uterine hypoplasia and growth retardation after weaning. Nat Genet 1997;16:391-6.

13. Daftary GS, Taylor HS. Endocrine regulation of HOX genes. Endocr Rev 2006;27:331-55.

14. Daftary GS, Taylor HS. Implantation in the human: the role of HOX genes. Semin Reprod Med 2000;18:311-20.

15. Kinuta K, Tanaka H, Moriwake T, et al. Vitamin D is an important factor in estrogen biosynthesis of both female and male gonads. Endocrinology 2000;141: 1317-24.

16. Aleyasin A, Hosseini MA, Mahdavi A, et al. Predictive value of the level of vitamin D in follicular fluid on the outcome of assisted reproductive technology. Eur 7 Obstet Gynecol Reprod Biol 2011;159:132-7.

17. Lagunova Z, Porojnicu AC, Lindberg F, et al. The dependency of vitamin D status on body mass index, gender, age and season. Anticancer Res 2009;29:3713-20.

Affiliations: Department of Obstetrics and Gynecology (Garbedian), Mount Sinai Hospital, University of Toronto; Faculty of Medicine (Boggild), University of Toronto; Samuel Lunenfeld Research Institute
(Moody), Mount Sinai Hospital; Department of Obstetrics and Gynecology (Liu), Mount Sinai Hospital, University of Toronto, Toronto, Ont.

Contributors: Kimberley Garbedian, Kimberly Liu and Joel Moody contributed to the study design and data analysis. Data collection was performed by Kimberley Garbedian and Miranda Boggild. The initial draft of the manuscript was written by Kimberley Garbedian, and all authors contributed to the revisions and gave final approval of the version submitted for publication.

Funding: Kimberely Garbedian and Kimberly Liu received research funding from the Centre for Fertility and Reproductive Health's Fellowship Research Fund to support this study.

Supplemental information: For reviewer comments and the original submission of this manuscript, please see www.cmajopen.ca/content/1/2 /E77/suppl/DC1 